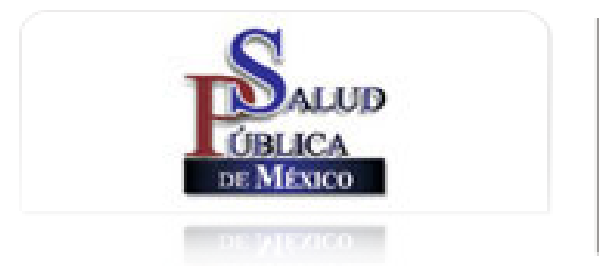

Salud Pública de México

ISSN: 0036-3634

spm@insp.mx

Instituto Nacional de Salud Pública

México

López-Hernández, Karla M; Pardío-Sedas, Violeta T; Williams, José de Jesús Evaluación del riesgo microbiológico a Vibrio spp. en alimentos de origen marino en México

Salud Pública de México, vol. 56, núm. 3, mayo-junio, 2014, pp. 295-301

Instituto Nacional de Salud Pública

Cuernavaca, México

Disponible en: http://www.redalyc.org/articulo.oa?id=10631164009

- Cómo citar el artículo

- Número completo

- Más información del artículo

- Página de la revista en redalyc.org

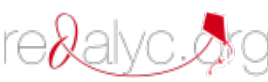

Sistema de Información Científica

Red de Revistas Científicas de América Latina, el Caribe, España y Portugal

Proyecto académico sin fines de lucro, desarrollado bajo la iniciativa de acceso abierto 


\title{
Evaluación del riesgo microbiológico a Vibrio spp. en alimentos de origen marino en México
}

\author{
Karla M López-Hernández, M en SAN, ${ }^{(1)}$ Violeta T Pardío-Sedas, D en $C$, ${ }^{(I)}$ José de Jesús Williams, D en C. ${ }^{(2)}$
}

\section{López-Hernández KM, Pardío-Sedas VT, Williams JJ. Evaluación del riesgo microbiológico a Vibrio spp. en alimentos de origen marino en México.} Salud Publica Mex 2014;56:295-30I.

\section{Resumen}

Las enfermedades transmitidas por los alimentos son uno de los mayores problemas de salud pública que actualmente existen. La evaluación del riesgo microbiológico es un proceso utilizado para examinar los peligros ocultos en los alimentos, la probabilidad de exposición a éstos y su impacto en la salud pública. La evaluación del riesgo se realiza en cuatro fases: identificación del peligro, caracterización del peligro, evaluación de la exposición y caracterización del riesgo. De acuerdo con el proceso/resultado, las evaluaciones de riesgo microbiológico se clasifican en dos categorías: cualitativa y cuantitativa. La presente revisión pretende enmarcar la importancia de implementar estas evaluaciones en alimentos de origen marino que son consumidos crudos, fortaleciendo así el acceso a los alimentos inocuos y de buena calidad para beneficio del consumidor, y la necesidad de evaluaciones de riesgo microbiológico que hay en México.

Palabras clave: Vibrio spp.; alimentos marinos; seguridad alimentaria; enfermedad; México

\author{
López-Hernández KM, Pardío-Sedas VT, Williams JJ. \\ Microbial risk assessment \\ of Vibrio spp. in seafood products in Mexico. \\ Salud Publica Mex 2014;56:295-30I.
}

\begin{abstract}
Food-borne diseases are among the major public health problems that currently exist. Microbiological risk assessment is a process used to evaluate the hidden hazards in food, the likelihood of exposure to these hazards and their impact on public health. Risk assessment is performed in four steps: hazard identification, hazard characterization, assessment of exposure and risk characterization.According to the process/ response microbial risk assessment is classified in two categories, qualitative and quantitative. The aim of this review is to underline the importance of implementing assessments in seafood that is usually consumed raw, strengthening access to good quality and safe food for the consumer's benefit and to stress the necessity of microbiological risks assessments in Mexico.
\end{abstract}

Key words: Vibrio spp.; seafood; food safety; disease; Mexico
$\mathrm{L}$ a industrialización del sector alimentario y el desaurollo del comercio internacional han posibilitado la distribución de los alimentos internacionalmente. Los peligros biológicos, químicos y físicos pueden ahora, por tanto, alcanzar poblaciones con distinta susceptibilidad y diferentes patrones de consumo con el consecuente riesgo sobre la salud. ${ }^{1}$ Debido a que los riesgos a la salud humana trasmitidos por los alimentos pueden deberse a causas de orden biológico, químico o físico, los riesgos por peligros microbiológicos constituyen un problema grave e inminente. Por lo tanto, se ha establecido y considerado al análisis de riesgos (AR)

(I) Programa de Maestría en Ciencia Animal, Facultad de Medicina Veterinaria y Zootecnia, Universidad Veracruzana. Veracruz, Veracruz, México.

(2) Dirección General de Desarrollo Académico, Edificio Central-Rectoría, Universidad Autónoma de Yucatán. Mérida, Yucatán, México.

Fecha de recibido: 6 de noviembre de 2013 - Fecha de aceptado: 19 de febrero de 2014 Autora de correspondencia: Dra.Violeta T. Pardío Sedas. Facultad de Medicina Veterinaria y Zootecnia, Universidad Veracruzana. Av. Miguel Ángel de Quevedo s/n, Col. UnidadVeracruzana. 917I0 Veracruz,Veracruz, México. Correo electrónico:vpardio@uv.mx,vpardio@yahoo.com.mx 
como un sistema de gestión del riesgo en el desarrollo de políticas de seguridad alimentaria para gobiernos y organizaciones internacionales. ${ }^{2}$

Según la Organización de las Naciones Unidas para la Alimentación y la Agricultura y la Organización Mundial de la Salud (FAO/OMS), el AR se compone de tres elementos: evaluación del riesgo (ER), gestión del riesgo (GR) y comunicación del riesgo (CR). ${ }^{3}$ La ER es la evaluación científica de un conocido o potencial efecto adverso contra la salud, resultado de la exposición humana a un peligro transmitido por los alimentos, e incluye la evaluación cuantitativa del riesgo (ECR) que ofrece una expresión numérica y cualitativa del riesgo, así como la incertidumbre asociada. ${ }^{1}$ De ahí que el riesgo microbiológico se exprese como la probabilidad de adquirir una enfermedad transmitida por alimentos (ETA). 4,5 La GR es definida como el "proceso de ponderar las distintas políticas posibles a la luz de los resultados de la evaluación del riesgo y, si procede, elegir y aplicar opciones de control apropiadas, incluidas las medidas reglamentarias". ${ }^{6}$ El último componente es la CR, definida como "el intercambio de información y opiniones sobre el riesgo entre los evaluadores del riesgo, los encargados de la gestión del mismo, los consumidores, la industria y otros interesados".7,8 Estos tres componentes deben estar separados funcionalmente para evitar cualquier tipo de conflicto de intereses. Sin embargo, debe considerarse que la interacción entre asesor y gestor del riesgo es esencial en su aplicación práctica. ${ }^{1}$ Más allá de definir lo que es el análisis de riesgo, la presente revisión pretende proporcionar un punto de referencia del estado actual en las evaluaciones del riesgo microbiológico (ERM) en México.

\section{Clasificación y principios generales de la evaluación del riesgo microbiológico}

En 1995, la Organización de las Naciones Unidas y la Organización Mundial de la Salud introdujeron el concepto de AR como un esquema sistematizado sobre el que se debe fundamentar el desarrollo de estándares alimentarios a escala nacional e internacional. ${ }^{3}$ La base científica del AR se aborda en la etapa de ER, cuyos resultados sustentan las posibles decisiones y políticas nacionales e internacionales en relación con un peligro o riesgo alimentario determinado. El beneficio del proceso estructurado de ER radica en la síntesis de datos e información, en representar relaciones complejas y describir la probabilidad y gravedad de las reacciones adversas, e informar el proceso de toma de decisiones. La ERM se clasifica en dos categorías: cualitativa y cuantitativa. En la cualitativa el riesgo es descrito como la probabilidad de enfermedad mientras que en la cuantitativa se expresa como el número predecible de casos de enfermedad. La ERM cuantitativa provee expresiones numéricas de riesgos para evaluar la probabilidad de efectos adversos en la salud humana debido a la exposición a microrganismos patógenos. $4,8,9$

\section{Proceso de la evaluación del riesgo microbiológico}

La ERM es el proceso que permite la estimación de la probabilidad y severidad de un resultado en particular, llamada riesgo estimado. La Comisión Mixta de la FAO/ OMS del Codex Alimentarius la define como un proceso con bases científicas que consta de cuatro fases: ${ }^{4,5,10-12}$

1. Identificación del peligro. Es un proceso cualitativo que consiste en identificar al microorganismo y/o sus toxinas que pueden asociarse con una enfermedad en particular. La información sobre los peligros puede obtenerse de la literatura científica básica y estudios clínicos, epidemiológicos y de vigilancia, considerando que se ha incrementado la identificación de peligros con técnicas moleculares que permiten la detección de patógenos difícilmente detectables por métodos convencionales.

2. Caracterización del peligro. Su componente primario es la relación dosis/respuesta, definida como el análisis de la relación entre la magnitud de la exposición a un agente biológico y la gravedad y/o frecuencia de la asociación a efectos adversos para la salud. La caracterización del peligro provee una descripción cualitativa o cuantitativa de la gravedad o duración de efectos adversos que pueden resultar de la ingestión de un microrganismo o toxina presente en los alimentos. La relación dosis / respuesta puede derivarse de investigaciones epidemiológicas.

3. Evaluación de la exposición. En esta fase se estima el nivel de patógenos o sus toxinas y la probabilidad de su presencia en un alimento al tiempo de su consumo, desde la producción al consumo. La exposición es básicamente una función de dos componentes: (a) la concentración del patógeno en el alimento y (b) la cantidad de alimento consumido, obteniendo una evaluación de la exposición humana al peligro. Los factores considerados importantes en la medición de la exposición son los patrones de consumo (el tamaño de la porción y la frecuencia de consumo) típicamente relacionados con factores socioeconómicos y culturales, étnicos, estacionalidad, edad y sexo, diferencias regionales, preferencias y conductas del consumidor. 
4. Caracterización del riesgo. Representa la integración de las determinaciones resultantes de las fases anteriores a fin de obtener una estimación del riesgo, proporcionando una estimación cualitativa y cuantitativa de la probabilidad y gravedad de los efectos adversos que podrían presentarse en una población dada. El resultado final es la estimación o predicción de enfermedades asociadas con un microrganismo particular.

Las evaluaciones del riesgo microbiológico en alimentos se han adaptado a partir del sistema de evaluación de riesgos químicos de la Academia Nacional de Ciencias de los Estados Unidos, aplicado por la Agencia de Protección Ambiental (US Environmental Protection Agency - USEPA) desde la década de los setenta y, debido a su complejidad y altos costos, son realizadas únicamente por organismos gubernamentales u organizaciones internacionales en los Estados Unidos, Canadá y la Unión Europea. ${ }^{13}$ En este contexto, la Unión Europea (UE) publicó el Libro Blanco sobre Seguridad Alimentaria, creó el Organismo Alimentario Europeo y la agencia independiente Autoridad Europea de Seguridad Alimentaria (EFSA, por sus siglas en inglés) para asegurar la protección al consumidor y mantener la confianza en el suministro alimentario, abarcando la seguridad de alimentos y piensos, la sanidad, el bienestar animal y la sanidad vegetal. ${ }^{14,15}$

\section{Evaluación del riesgo microbiológico en productos marinos}

Los productos marinos son altamente perecederos ya que fácilmente se contaminan con microorganismos patógenos, como los moluscos bivalvos, y debido a que filtran grandes cantidades de agua, bioacumulan sustancias tóxicas y microorganismos presentes en su entorno, incluyendo miembros de la familia Vibrionaceae como Vibrio cholerae, Vibrio parahaemolyticus y Vibrio vulnificus, cuya incidencia sigue constituyendo uno de los problemas de salud pública en el país y a nivel mundial debido al creciente consumo de mariscos y pescados crudos, mal cocidos y contaminados. ${ }^{16,17}$

La evaluación de riesgos preliminar de la Food and Drug Administration (FDA) de impactos sobre la salud pública a $V$. parahaemolyticus (FDA-VPRA) señala las conexiones entre la prevalencia de $V$. parahaemolyticus en ostiones crudos y la temperatura de las aguas de recolección, mostrada en la figura 1. En este modelo para el módulo de recolección, la temperatura del agua es el factor principal que determina el número inicial de $V$. parahaemolyticus en los ostiones. Las variaciones de temperatura y salinidad regionales y estacionales permiten el análisis plurianual. El módulo postcosecha establece el papel que desempeñan la elaboración y la manipulación postcosecha en el número de V. parahaemolyticus patógenos en el consumo. El término "Vp/g en la postcosecha" es el resultado del modelo de recolección. Los datos sobre el tiempo que permanecen los ostiones fuera del agua y la temperatura del aire se utilizan para predecir el crecimiento de $V$. parahaemolyticus en los ostiones. Los niveles de $V$. parahaemolyticus disminuyen durante el almacenamiento refrigerado y, por tanto, el tiempo de almacenamiento es un factor que influye en el número de $V$. parahaemolyticus. En el módulo de consumo, el valor de "Vp/g en el consumo" indica el resultado del módulo postcosecha. Para obtener la dosis ingerida, la densidad se multiplica por el número de ostiones por ración y el peso de los ostiones. Dicha dosis ingerida se utiliza en la relación dosis-respuesta para calcular el riesgo de enfermedad asociado con el consumo de una ración de ostiones. ${ }^{18}$

El modelo resulta aplicable a otros patógenos y, debido al riesgo microbiológico presente en alimentos marinos, se ha empleado en diferentes combinaciones de patógeno/ producto en otros países. ${ }^{17,19,20}$ En el cuadro I se presentan algunos reportes internacionales de ERM realizadas para Vibrio spp en mariscos crudos.

\section{Evaluación de riesgo microbiológico en México}

La Secretaría de Salud, a través de la Dirección General de Control Sanitario de Bienes y Servicios publicó en 1993 el Manual de aplicación del análisis de riesgos, identificación y control de puntos críticos para garantizar la seguridad de los alimentos con énfasis en la prevención, delegando la responsabilidad de la seguridad de los productos a las empresas que los elaboran, de manera que al conocer los puntos críticos de su proceso los controlen. ${ }^{21,22}$ Cabe considerar que las zoonosis y contaminaciones por gérmenes patógenos en los animales son importantes por la gravedad de las infecciones que producen en el hombre, de ahí la importancia de determinar la calidad sanitaria de los alimentos de mayor consumo por la población.

En México se han realizado algunas evaluaciones de la calidad sanitaria de productos marinos. Se reportó la prevalencia de Vibrio spp. en tres puestos de venta de mariscos en el norte de la Ciudad de México durante mayo de 2004. Del total de 48 muestras, 18 fueron ostiones crudos, 12 de "ceviche" de pescado (crudo marinado en jugo de limón) y 18 cocteles de camarón (ligeramente hervidos). Las muestras de ostión (77.7\%), de ceviche $(33.3 \%)$ y de cocteles de camarón (16.6\%) resultaron contaminadas con Vibrio cholerae no-O1/ no-O139 y Vibrio parahaemolyticus, revelando así el riesgo para la salud 
A

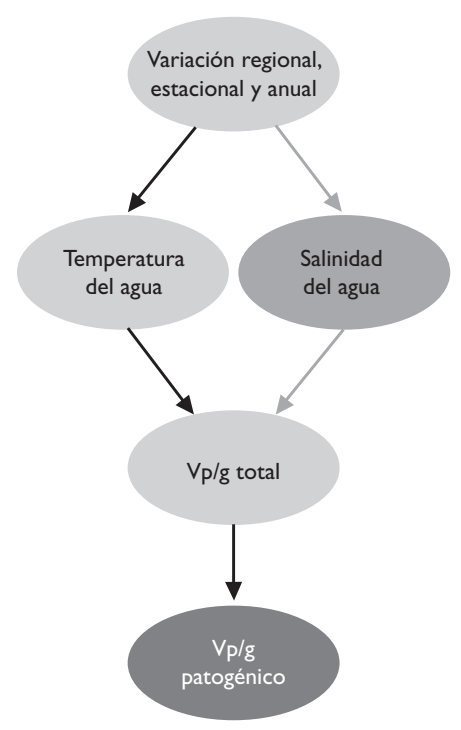

B

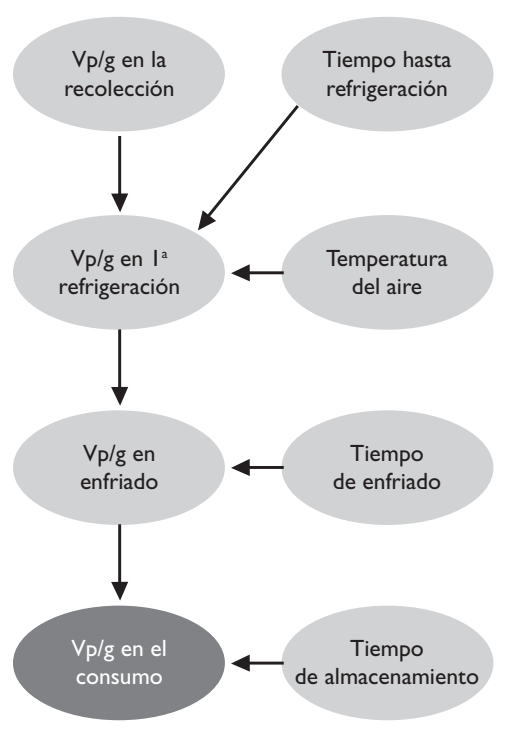

C

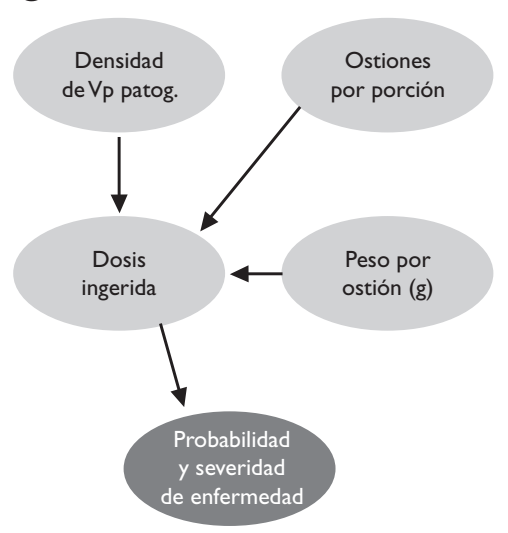

Figura I. Módulos de recolección (A), postrecolección (B) y de consumo (C) del modelo FDA-VPRA.18 Evaluación del riesgo microbiológico a Vibrio spp en alimentos de origen marino en México

\section{Cuadro I \\ Reportes internacionales de evaluaciones de riesgo microbiológico de Vibrio cholerae, Vibrio parahaemolyticus y Vibrio vulnificus realizadas en mariscos crudos}

Patógeno en producto de interés/procedencia Fuente (año de publicación)

Vibrio spp. en mariscos de: Japón, Nueva Zelandia, Canadá, Australia, Tailandia y Estados Unidos

Vibrio parahaemolyticus en moluscos de: Costas del Golfo,Atlántico y Pacífico, Estados Unidos

Vibrio cholerae OI y OI39 patogénico en camarones de agua templada para exportación de Estados Unidos
FAO/WHO* $(200 I)^{19}$

FDA $^{\ddagger}$, EUA $(2004)^{19}$

FAO/WHO $(2002)^{20}$
Resumen de resultados

Acciones como el lavado a fondo y la preparación segura de mariscos fueron identificadas como las mitigaciones clave para reducir los riesgos de enfermedad causada por vibrios en estos alimentos.

Se encontró que la temperatura del agua en el momento de la recolección era la principal influencia en los niveles iniciales de V. parahaemolyticus en ostiones. Había una significativa reducción de las probabilidades de enfermedad cuando los ostiones eran enfriados inmediatamente luego de la recolección.

Se observó que sólo eran agentes causales del cólera las cepas que producen la toxina del cólera y que pertenecen a los serotipos OI y OI39. Las reducciones logarítmicas del número de organismos coleragénicos se producen durante el lavado, la congelación y la cocción. Es necesaria una dosis elevada de la cepa OI $\left(10^{6}\right)$ de V. cholerae coleragénica para causar la enfermedad por ingestión de $V$. cholerae con los alimentos. La evaluación de riesgos cualitativa (descriptiva) mostró que no existía un problema de salud pública asociado con el consumo de camarones importados de aguas templadas.

Vibrio parahaemolyticus en las almejas Anada- FAO/WHO (2002) ${ }^{20}$ ra granosa de Songkla, Tailandia

Se estimó que la posibilidad media de enfermedades anuales por consumo de almejas era de $9.18 \times 10^{10}$ por persona (se enferma aprox. I persona de I 000 $000000 \mathrm{al}$ año) y la posibilidad máxima era de $9.34 \times 10^{6}$ (se enferma aproximadamente I persona de 100000 al año).

Vibrio vulnificus en ostiones de Costas del FAO/WHO (2005) ${ }^{17}$

El riesgo medio de enfermedad debida a Vibrio vulnificus por ración o exposición basándose en informes de casos y estadísticas de consumo para invierno, primavera, verano y otoño fue de $1.4 \times 10^{-6}, 2.8 \times 10^{-5}, 4.9 \times 10^{-5}$ y $1.9 \times 10^{-5}$ respectivamente.

\footnotetext{
* FAO/WHO: Food and Agriculture Organization of the United Nations/World Health Organization
}

₹ FDA: US Food and Drug Administration 
que representa su consumo. ${ }^{23}$ En otro estudio realizado en 260 muestras de almejas obtenidas en un ciclo anual en el estado de Veracruz, se aislaron $V$. cholerae O1 Inaba patogénico $(7 \%)$ y $V$. cholerae no $\mathrm{O} 1(21.5 \%)$. Aunque en todo el ciclo se obtuvieron aislamientos, la mayor contaminación se presentó en los meses de mayo, junio, julio y agosto. ${ }^{24}$

La ocurrencia de los vibrios patógenos en los productos acuícolas está influenciada por las características fisicoquímicas del ambiente acuático y diversas variables ambientales que pueden influir en su compleja ecología, incrementando el riesgo. ${ }^{25}$ En el Golfo de México se ha reportado la presencia de Vibrio cholerae O1 Inaba (ct $x+)$ durante la época de lluvias (julio-septiembre) en 100\% de las muestras de ostiones (Crassostrea virginica) extraídos de las lagunas La Mancha y Alvarado, Veracruz. ${ }^{26}$ Los estudios en el ostión (C. virginica) extraído del sistema lagunar Mandinga, Veracruz, durante los años 2008-2012, indican la influencia de la época estacional en la presencia de genes patogénicos. El análisis de exposición a $V$. cholerae no-O/no-139 en el ostión cosechado de esta laguna mostró un mayor riesgo relativo (0.6) relacionado con la temporada de estiaje, indicando que existen más probabilidades de que una persona se enferme por el consumo de ostiones contaminados durante los meses de marzo, abril, mayo y junio. ${ }^{27,28}$ Con respecto a la patogenicidad, $25 \%$ de 200 UFC/g ambientales de V. cholerae no-O1/ no-O139 en muestras de ostiones presentaron el gen ch $x A$ patogénico. ${ }^{29}$ Asimismo, se han reportado varios casos de intoxicación alimentaria en Victoria, Tampico y Matamoros, Tamaulipas, México, debido a la ocurrencia de $V$. parahaemolyticus en muestras de ostiones. ${ }^{30,31}$

El primer estudio y reporte de la evaluación de la exposición y del riesgo relacionado con la presencia y variación estacional de Vibrio parahaemolyticus y Vibrio vulnificus voha+ en México se realizó en ostiones extraídos de la laguna de Mandinga, Veracruz, que reportó el incremento significativo $(p<0.05)$ del riesgo de exposición (1.81) a V. vulnificus para los consumidores de ostión en verano, mientras que para $V$. parahaemolyticus ( $t l h+$ ) fue mayor $(p<0.05)$ en la época de primavera (marzo, abril y mayo) con 1.21 y 1.03 para $V$. parahaemolyticus $(t l h+/ t d h+/ t r h-)$ patogénico. ${ }^{32,33}$

En el Pacífico mexicano, el primer brote de gastroenteritis causada por cepas pandémicas de $V$. parahaemolyticus O3:K6 en México fue reportado en el sur del estado de Sinaloa en septiembre de 2004 que afectó a más de 1250 personas debido al consumo de camarones crudos o poco cocidos del sistema lagunar Huizache-Caimanero contaminados con V. parahamolyticus $(t d h+/ t r h-),(t d h+/ t r h+)$ y O3:K6 patógenicos. ${ }^{34}$ En el mismo estado, entre 2004-2010 se reportaron más de 1230 casos de gastroenteritis asociados con el consumo de camarones. Los resultados demostraron que la cepa pandémica $V$. parahaemolyticus O3:K6 ambiental fue la causante de $79 \%$ de los casos reportados. ${ }^{35}$

\section{Discusión}

A la luz de la dependencia cada vez mayor del hombre de los ecosistemas marinos, la evolución y la emergencia continua en los últimos 100 años de nuevas variantes patogénicas de Vibrio spp representa uno de los mayores retos para los gobiernos en el intento de controlar y manejar las enfermedades infecciosas que pueden evolucionar a septicemia y muerte (DL50 $>50 \%$ ), principalmente en individuos con padecimientos crónico-degenerativos. Considerando que la ecología microbiana de estos patógenos varía entre las regiones del mismo país, que la infección por estos patógenos está subdiagnosticada y que el consumo de mariscos en México está muy extendido, es importante prevenir potenciales brotes infecciosos. Es necesario resaltar que la ERM proporciona una imagen más detallada de cómo se generan estos riesgos. ${ }^{36} \mathrm{Sin}$ embargo, en México existe poco desarrollo en materia de AR. Los estudios en México demuestran la presencia de patógenos humanos del género Vibrio en alimentos marinos consumidos crudos y poco cocidos procedentes de ambas costas, del Golfo de México y del Océano Pacífico; sin embargo, la mayoría de estas investigaciones tienen un enfoque sanitario y epidemiológico, a diferencia de lo que ocurre en otros países europeos, en Japón o en EUA, donde, además de utilizar las normas internacionales establecidas, también realizan evaluaciones con normas desarrolladas por empresas con alcance internacional o, inclusive, por agencias privadas como EFSA. ${ }^{37}$

La ERM de alimentos marinos permitiría conocer la probabilidad de los efectos adversos en la salud humana (enfermedades diarreicas, hospitalizaciones y muertes) por el consumo de alimentos contaminados, así como la mejor opción para reducir el riesgo, por lo que las acciones a implementar deberán incluir monitoreos obligatorios para generar información de las condiciones favorables a un brote para alertar y prevenir la pesca o el consumo, enfatizar el control del producto en el punto de captura, adoptar un buen manejo sanitario postcosecha, detectar un incremento de la virulencia, así como evaluar el riesgo del crecimiento postcosecha en tiempo real, es decir, la sobrevivencia y transporte del patógeno específico durante la etapa de distribución, venta y consumo en los establecimientos dedicados a su venta, lo cual permitiría conocer incluso si otros factores como el transporte, el almacenamiento (cadena fría) y la contaminación cruzada están influenciando los niveles de contaminación y ésta no es causada únicamente por la contaminación de origen presente 
en el alimento. Es indispensable generar iniciativas regulatorias que sean adoptadas para limitar las pérdidas causadas por los patógenos y evitar la transmisión de enfermedades, e informar a los consumidores de los riesgos de consumir pescado y mariscos crudos, mal cocidos o marinados aun cuando las medidas preventivas se hayan aplicado. Estas acciones ayudarían a mitigar el impacto económico, social y ambiental en el país, incrementando la oferta de alimentos inocuos de calidad reconocida internacionalmente.

La implementación de ERM en alimentos debe ser congruente con los objetivos generales de salud pública establecidos por las autoridades gubernamentales, en apoyo al sector agropecuario, para favorecer y facilitar las exportaciones y la competitividad internacional. Para ello, es imprescindible contar en el país con sistemas de vigilancia epidemiológica eficaces y un seguimiento de los brotes de enfermedades con una visión integradora y realista para poder hacer frente a los retos que conlleva. Es importante reconocer que el rumbo que México ha tomado en materia política, económica y comercial hace necesaria la aplicación de ERM en el marco de las políticas de salud pública para la obtención de alimentos de calidad en beneficio del consumidor.

\section{Conclusiones}

De acuerdo con los estudios realizados en México, el riesgo de exposición a Vibrio patogénico con potencial pandémico en diversos mariscos está demostrado, representando un riesgo significativo para el sector pesquero y la salud pública. El potencial zoonótico de estos patógenos requiere ser reconocido para poder prevenir las condiciones que promueven su abundancia e implementar soluciones de control.

La complejidad del problema requiere del establecimiento de monitoreos de vigilancia y de investigación sobre su ecología y los factores que favorecen su ocurrencia en los productos marinos, así como de la implementación de métodos estandarizados que faciliten su detección rápida y segura para producir datos confiables y crear bases de datos que permitan la evaluación del riesgo para la población humana asociada con la utilización de los ambientes costeros y el consumo de alimentos marinos. De ahí que sea impostergable la implementación de ERM para predecir el riesgo a partir de la exposición, prevenir brotes asociados a los Vibrios spp. y disminuir el impacto de las enfermedades de transmisión por alimentos.

En la presente revisión se mencionaron algunas evaluaciones de riesgo microbiológico a Vibrio spp. en productos marinos realizadas en espera de que este documento promueva la reflexión y consideración de diversas instancias y sea posible que se realicen evaluaciones de riesgo microbiológico en México de manera normativa, tomando como base la importancia del acceso a los alimentos inocuos y de buena calidad a fin de salvaguardar la salud pública.

Declaración de conflicto de intereses. Los autores declararon no tener conflicto de intereses.

\section{Referencias}

I. Pérez RF. Evaluación cuantitativa del riesgo microbiano en productos cárnicos cocidos: Modelos de contaminación cruzada y su impacto sobre la gestión del riesgo (tesis). Argentina: Universidad de Córdoba, 2006.

2. Food and Agriculture Organization of the United Nations/World Health Organization (FAO/WHO). Food safety risk analysis:A guide for national food safety authorities. Food and Nutrition paper Series; 2006:87. 3. Food Agriculture Organization/World Health Organization (FAO/ $\mathrm{WHO}$ ).Application of risk analysis to food standards. Report of the joint FAO/WHO expert consultation; 1995 march 13- I7; Geneva.

4. Schroeder CM, Jensen E, Miliotis MD, Dennis SB, Morgan MK.

Microbial risk assessment. En: Shabbir Simjee ed. Foodborne Diseases. Hampshire, UK: Humana Press Inc, 2007:435-456.

5. Food and Agriculture Organization of the United Nations/World Health Organization (FAO/WHO). Risk Characterization of Microbiological Hazards in Food. Microbiological Risk Assessment Series; 2009: 17. 6. Food Agriculture Organization/World Health Organization (FAO/ WHO). Codex Alimentarius Commission. Procedural manual. Twelfth Edition: Procedures for the elaboration of codex standards and related texts. Joint FAO/WHO food standards programme, codex alimentarius commission. Roma: FAO/WHO, 200I.

7. Food Agriculture Organization/World Health Organization (FAO/ WHO).Application of risk communication to food standards and food safety. Report of the joint FAO/WHO expert consultation; 1998 february 2-6; Rome.

8. Miliotis M. Role of microbial risk assessment in food safety. S Afr Med J 2007;97(I I):I2II-I2I 4

9.Vose D. Risk analysis: a quantitative guide. Chichcstcr, UK: John Wiley \& Sons, 2000: II8-159.

10. Skinner R. Microbiological Risk Assessment and Public Health. International conference on risk assessment; London, UK: 1992 oct 5-9.

I I. Barraj LM, Petersen BJ. Food consumption data in Microbiological risk assessment. J Food Protect 2004;67(9): 1972-1976.

12. Food and Agriculture Organization of the United Nations/World Health Organization (FAO/WHO). Exposure assessment of Microbiological hazards in food. Guidelines. Microbiological Risk Assessment Series, 7. Roma: FAO, 2008.

13. Schothorst MV. Microbiological risk assessment of foods in international trade. Safety Science 2002;40( I-4):359-382.

14. Commission of the European Communities (CEC). White Paper on Food Safety. COM (1999) 719 final. Brussels: CEC, 2000.

I5.Agencia Española de Seguridad Alimentaria y Nutrición (AESAN). AESAN: Punto de contacto de EFSA, 2013 [consultado en 20I3]. Disponible en: http://www.aesan.msc.es/AESAN/web/punto_focal_efsa/ punto_focal_efsa.shtml

16. Baris BE, Colak H. The microbiological quality of stuffed mussels (Midye dolma) sold in Istanbul. Br Food J 2008; I I0( I I): 1079- 1089 17. Food and Agriculture Organization of the United Nations/World Health Organization (FAO/WHO). Risk assessment of Vibrio vulnificus in raw oysters. Interpretative summary and technical report. Microbiological risk assessment Series, 2005:8. 
18. Food and Agriculture Organization/World Health Organization (FAO/ WHO). Joint FAO/WHO Expert consultation on risk assessment Microbiological hazards in foods; Geneva, Switzerland; 2001 july 23-27.

19. Forsythe SJ.The Microbiology of safe food. 2a ed. lowa:Wiley-Blackwell, 2010:166-192.

20. Food and Agriculture Organization of the United Nations/World Health Organization (FAO/WHO). Risk assessment of Campylobacter spp in broiler chickens and Vibrio spp in seafood: Report of a Joint FAO/WHO Expert consultation. FAO Food and Nutrition paper Series, 2002:75. 2I. Zarco GE. Manual de aplicación del análisis de riesgos, identificación y control de puntos críticos, 1993. Secretaría de Salud, México, D.F. I-2 [consultado en 2013]. Disponible en:www.salud.gob.mx/unidades/cdi/ documentos/DOCSAL2628.doc

22. Centro de Investigación en Alimentación y Desarrollo (CIAD). Foro FAO-CIAD sobre evaluación de riesgos microbiológicos en alimentos; 2002 febrero 7 y 8; Hermosillo, Sonora.

23. Estrada-García T, López-Saucedo C, Arévalo C, Flores-Romo L, Luna O, Pérez-Martínez I. Street-vended seafood: a risk for foodborne diseases in Mexico. Lancet Infect Dis 2005;5:59-70.

24. Quiñones REI,Vázques SC, Pedroche FF, Moreno SL, Rodas SOR. Presencia de los géneros Vibrio y salmonella y detección de coliformes fecales en almejas del Golfo de México. Hidrobiológica 2000;10(2): I31-138. 25. Pardío SVT. Influence of environmental factors on the presence of Vibrio cholerae in the marine environment: a climate link.J Infect Developing Countries 2007; I(3):224-24I.

26. Castañeda-Chávez M, Pardío SV, Orrantia E, Lango-Reynoso F. Influence of water temperature and salinity on seasonal occurrences of Vibrio cholerae and enteric bacteria in oyster-producing areas of Veracruz, México. Mar Poll Bull 2005;50(I2): 164|-1648.

27. Pardío V, López K, Uscanga R, Márquez O, Quintero L, Cisneros N, et al. Vibrio cholerae and enteric bacteria in American oyster (Crassostrea virginica) from the harvesting areas of the Mandinga Lagoon (Veracruz, México). Institute of Food Technologists Annual Meeting and Food Expo; 2008 junio 28- julio I; Nueva Orleans, LO, EUA.

28. Martínez RJF. Ocurrencia estacional de Vibrio spp en moluscos bivalvos (Crassostrea virginica) procedentes de Mandinga, Veracruz (tesis). Veracruz, México: Facultad de Medicina Veterinaria y Zootecnia-Universidad Veracruzana, 2011.
29. Pardío V, Lizárraga-Partida ML, López KM, Gómez-Gil B,Wong I, Méndez E, et al. Detection of Cholix toxin from Vibrio cholera non-OI/ non-OI39 in oysters (Crassostrea virginica) harvested from Mandinga Lagoon, México. Institute of Food Technologists, Annual Meeting and Food Expo; 2012 june 25-28; Las Vegas, NV.

30. Charles-Hernández G, Cifuentes E, Rothenberg J. Environmental factors associated with the presence of Vibrio parahaemolyticus in sea products and the risk of food poisoning in communities bordering the Gulf of Mexico. Int J Environ Health Res 2006; 2(5):75-80.

3I. Ramírez-Martínez H, Charles-Hernandez G, Guerrero-Cumpean E, Chuc-Manzanilla BE, Becerra-Cruz EM. Posible foco endémico de Vibrio parahaemolyticus. Tamaulipas: Memorias, 2003.

32. Flores R, Pardío V, Lizárraga L, Martínez D, López K, Flores P, et al. Estimación del riesgo de infección por Vibrio vulnificus por el consumo de Crassostrea virginica extraído de Mandinga, Veracruz. XLIX Reunión Nacional de Investigación Pecuaria,Veracruz, 109; 2013 sept. 10-13; Boca del Río,Veracruz, México.

33. Rendón K, Pardío V, Lizárraga L, Flores P, Martínez D, López K, et al. Densidad total y patogénica de Vibrio parahaemolyticus en ostión americano (Crassostrea virginica) extraído de Mandinga,Veracruz y el riesgo asociado a su consumo. XLIX Reunión Nacional de Investigación Pecuaria, Veracruz, 109; 2013 sept. 10-13; Boca del Río,Veracruz, México. 34. Cabanillas-Beltrán H, Llausás-Magaña E, Romero R, Espinoza A, García-Gasca A, Nishibuchi M, et al. Outbreak of gastroenteritis caused by the pandemic Vibrio parahaemolyticus O3:K6 in Mexico. FEMS Microbiology Letters 2006;265:76-80.

35. Velázquez-Roman J, León-Sicairos N, Flores-Villaseñor H,VillafañaRauda S, Canizalez-Román A.Association of pandemic Vibrio parahaemolyticus O3:K6 present in the coastal environment of Northwest Mexico with cases of recurrent diarrea between 2004 and 2010.Appl Environ Microbiol 2012;78(6):1794-1803.

36. Hoffmann S. Ensuring Food Safety around the Globe:The many roles of risk analysis from risk ranking to Microbial risk assessment. Risk Analysis 2010;30(5):71I-714.

37. Wolff C. Private standards and the WTO Committee on Sanitary and Phytosanitary Measures. World Organization for Animal Health 2008;87-93. 